\title{
Physical Classification and Recent Developments of Active Noise Control Systems
}

\author{
M-W. Munir, Waleed Abdulla, Iman Ardekani*, Nitish Patel \\ Department of Electrical and Computer Engineering, The University of Auckland, New Zealand \\ *Department of Computer Science, Unitec Institute of Technology, New Zealand \\ mmun473@aucklanduni.ac.nz, (w.abdulla,nd.petal)@auckland.ac.nz, *iardekani@unitec.ac.nz
}

\begin{abstract}
The rapid growth of the industry has a major effect on the environmental noise pollution, and it ranks second to the air pollution that adversely affects the human health. Passive noise control techniques are impractical and very expensive for lowfrequency noises. To solve such acoustic problem active noise control has been studied since early 20th century. Active noise control is based on the principle of superposition: i.e., it mitigates the unwanted noise by generating an anti-noise having the same amplitude but opposite in phase. In this paper, we present the physical classification of the existing literature on the basis of both noise source and quiet zone characteristics. Examples are point to point, point to zone, zone to point and zone to zone. We focus on the developing trends of active noise control in the last decade and discuss latest add-on features and multi-channel active acoustic shielding for open windows.
\end{abstract}

\section{CCS Concepts}

- Hardware $\rightarrow$ Digital signal processing.

\section{Keywords}

Active noise control; Acoustic noise cancelling; Digital signal processing; Adaptive filtering.

\section{INTRODUCTION}

Environmental noise problem has become a more serious issue with the rapid growth of the transportation and industry. According to the WHO, $40 \%$ of the European population exposed to noise at a level of $55 \mathrm{~dB}$, and noise pollution alone causes 43000 hospital admissions per year [1]. The de facto acoustic noise mitigation techniques are based on the passive noise control that scatters and/or dampens acoustic waves such as sound mufflers, acoustic absorbers, and insulators. However, passive noise control techniques require bulky, costly materials and ineffective for lowfrequency noises. This is because at low frequency the acoustic wavelength becomes large as compared to the thickness of the typical acoustic absorber/insulator. To surpass these drawbacks is the major motivation towards active noise control. ANC has an existence from early 20th century, but after the development of adaptive filters [2-4] and powerful digital processors [5-8], it has gained significant research interest in the last two decades to reduce the low-frequency noises.

Permission to make digital or hard copies of all or part of this work for personal or classroom use is granted without fee provided that copies are not made or distributed for profit or commercial advantage and that copies bear this notice and the full citation on the first page. Copyrights for components of this work owned by others than ACM must be honored. Abstracting with credit is permitted. To copy otherwise, or republish, to post on servers or to redistribute to lists, requires prior specific permission and/or a fee. Request permissions from Permissions@acm.org. ICSPS 2017, November 27-30, 2017, Auckland, New Zealand

(C) 2017 Association for Computing Machinery.

ACM ISBN 978-1-4503-5384-7/17/11 ..\$15.00

https://doi.org/10.1145/3163080.3163099
Active noise control is based on the principle of superposition by generating the secondary noise of equal magnitude but opposite in phase of the undesired noise. The first experiment of superposition of sound was made by Lord Rayleigh in 1878 by the two electromechanically synchronized tuning forks [9]. The electro-acoustical study to mitigate the unwanted noise by exploiting the destructive interference of sound started in the 1930s $[10,11]$, and the first patent on active noise control utilizing a microphone and the electronically driven loudspeaker was granted to Paul Lueg in 1936 [12].

In practical applications, the performance of active noise control is mainly dependent on the accurate estimation of the phase, amplitude, and frequency of primary noise signal [13]. Since the characteristics of the acoustic noise source and physical environment are time varying. To cope with these variations the active noise control system must be adaptive. Burgess was the first to used adaptive filter for ANC [14]. The most common form of the adaptive filters are transversal filters. The transversal filters are adaptive by varying its weight factor using adaptive algorithms, of which the LMS algorithm is the most popular one. Widrow and Hoff introduced the LMS algorithm as an adaptive algorithm in 1959 [2].

To achieve viable noise attenuation at fast convergence rate ANC must be digital $[15,16]$. The development of advanced VLSI and digital signal processing systems help in implementing robust adaptive algorithms in real time. In late 20th century, Active noise control gained significant research interest, and a large number of sophisticated algorithms was developed and implemented in real time [17-19].

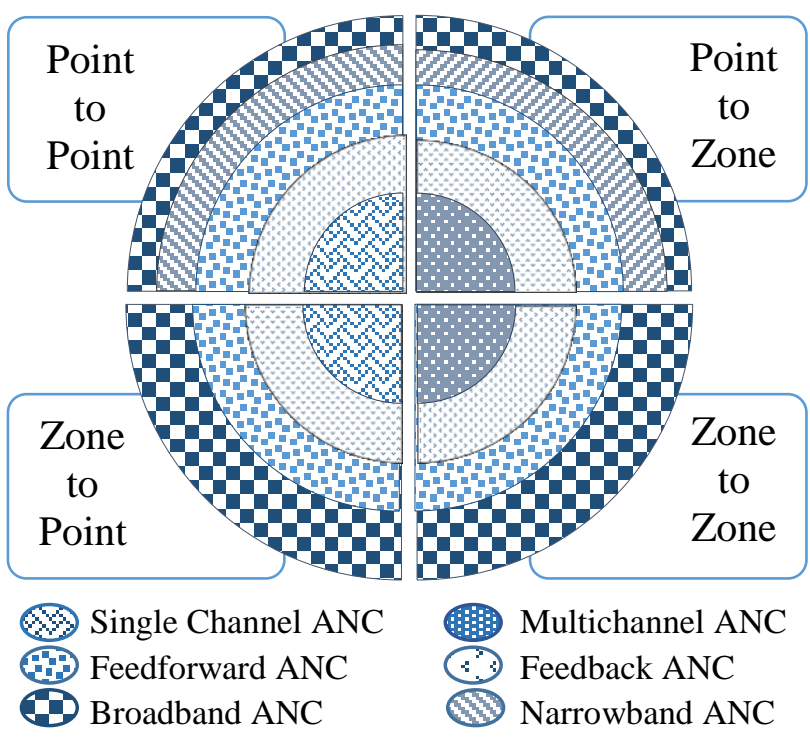

Figure 1. Physical Classification of Active Noise Control. 
Traditionally active noise control is classified into two categories such as local ANC and spatial ANC. In local ANC the target area is defined locally at some specific points in space. While in spatial ANC the aim is to achieve the noise reduction spatially in the large space. The spatial ANC seems more beneficial as compared to local ANC, but physical and practical constraint makes it more complicated for implementation. Similar to quiet zone the noise source also have two categories point source and spatial source. In Figure 1 we have classified these four terms on the basis of the both noise source and quiet zone characteristics.

\section{POINT TO POINT ANC}

The most efficient and simple model of active noise control systems is the point to point ANC, in which the noise source is positioned in a small area, and the targeted quiet zone is local.

\subsection{Feedforward ANC}

An acoustic duct is the best example of point to point ANC, and this is the most cultivated area of active noise control. Lueg's first patent of active noise control is based on this point to point duct model approach. A reference microphone is used to pick the acoustic noise signal, which is processed by an electronic system (phase inverter and delay), to generate an anti-noise with the secondary loudspeaker that is same in amplitude but opposite in phase of the primary noise. At the position of error microphone, these two noises cancel each other to form a local quiet zone. This model is called feedforward active noise control. To achieve the satisfying noise reduction, the electronic system must be adaptive to rectify the non-uniform amplitude and phase response of the amplifier, filter, and transducers. In 1981 Burgess implemented the digital adaptive filters in ANC acoustic duct [14] and later in 1984 frequency domain analysis of active acoustic duct was presented by Roure [20]. A practical system model for active attenuation in ducts was presented by Eriksson in 1988 [21]. The second-most important factor for satisfying the performance is the casualty conditions. After picking the reference signal, the controller takes some time to process and generate an anti-noise signal through the canceling loudspeaker. If the electrical delay from the reference microphone to the loudspeaker is larger than the acoustic delay, then it significantly degrades the performance of ANC system. However, it performs well in case of periodic and narrowband noises, but in the event of broadband noises, it is necessary to model the secondary path accurately to compensate the electrical delay and met the causality conditions. A large number of secondary path modeling techniques are available in the literature, of which Filtered-x LMS and variants are the most adopted. Morgan suggested the FxLMS algorithm by placing a secondary path estimate $\widehat{\boldsymbol{s}}(\mathrm{z})$ to the filter of reference signal $x(n)$ [22], the filtered reference signal $\widehat{\boldsymbol{x}}(n)$ used for the weight update of adaptive algorithm. Later the complete implementation of FxLMS was driven by Widrow [23]. The basic point to point single channel ANC system with FxLMS is outlined in Figure 2 [18]. It comprises a reference sensor to pick up the reference noise $x(n)$. This reference signal is processed by the adaptive filter $W(z)$ to generate the cancelling signal $y^{\prime}(n)$ driven by cancelling loudspeaker, where $y^{\prime}(n)=s(n)^{*} y(n)$ is the cancelling signal, * denotes linear convolution, and $s(n)$ is the impulse response of the secondary path $\mathrm{S}(\mathrm{z})$. Where $e(n)$ residual error pick up by the error microphone $e(n)=d(n)-y^{\prime}(n)$.

The standard FxLMS algorithm is summarized as:

$$
y(n)=\mathbf{w}^{T}(\mathrm{n}) \mathbf{x}(\mathrm{n})
$$

where $\quad \mathbf{w}(\mathrm{n})=\left[\begin{array}{lll}w_{0}(n) & w_{1}(n) & w_{L-1}(n)\end{array}\right]^{T}$

and $\quad \mathbf{x}(\mathrm{n})=\left[\begin{array}{lll}x(n) & x(n-1) & x(n-L+1)\end{array}\right]^{T}$

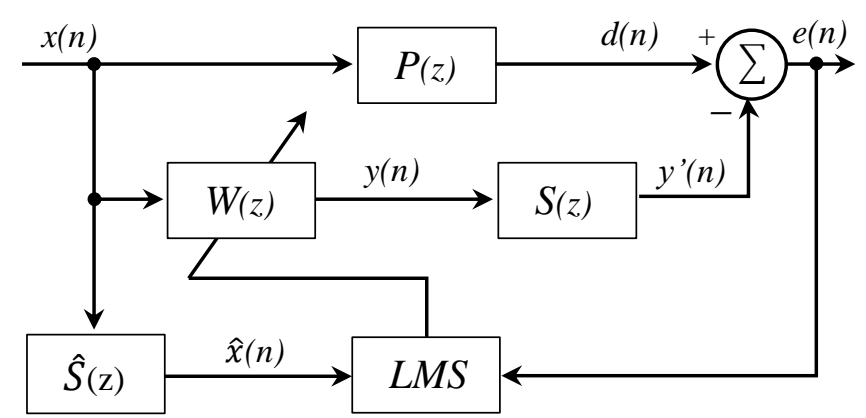

Figure 2. Block Diagram of Feedforward ANC System.

are the controller coefficients of length $\mathrm{L}$ and the input signal vectors, respectively.

$$
\hat{\mathbf{x}}(n)=\hat{s}(n) * \mathbf{x}(n)
$$

Where $\hat{x}(n)$ is the filtered reference signal of the input $x(n)$, and $\hat{s}(n)$ is the estimated impulse response of the secondary path. The FxLMS algorithm updates the coefficient vector as

$$
\mathbf{w}(n+1)=\mathbf{w}(n)+\mu \hat{\mathbf{x}}(n) e(n)
$$

where $\mu$ is the step size that determines the convergence speed.

The analyses of both perfect and imperfect secondary path estimation of feedforward active noise control system are presented in [24-26].

\subsection{Feedback ANC}

FxLMS based feedforward ANC system used two sensors, i.e., reference sensor and error sensor to attenuate the noise. In many real-world applications, the use of the reference sensor is not the perfect solution either from a microphone or any other type of non-acoustic sensors. These limitations promote the application of feedback active noise control system which only uses an error sensor. The block diagram of a feedback ANC system is shown in Figure 3.

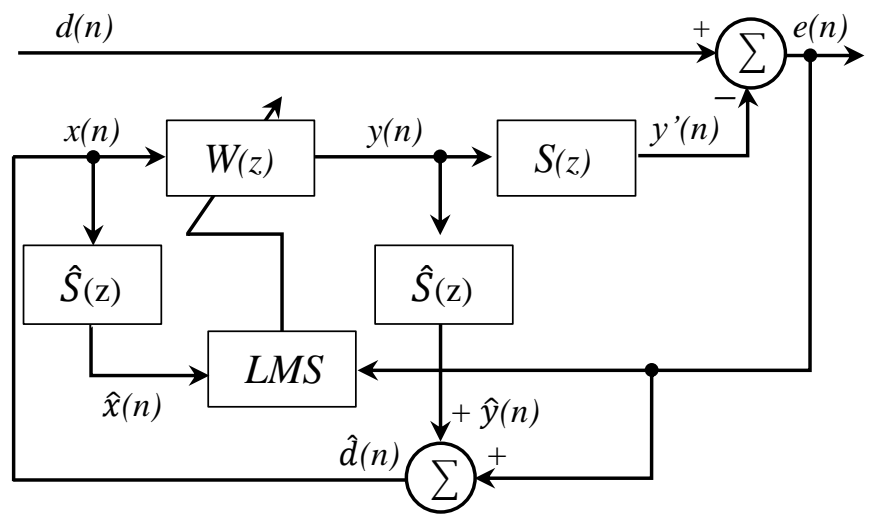

Figure 3. Block Diagram of Feedback ANC System.

The principle of the feedback active noise control system is using the measured error signal to synthesize the reference signal. The synthesized reference signal $\hat{d}(n)$ is the combination of $\hat{y}(n)$ and $e(n)$, because the reference signal for the next iteration is based on the current estimation of $d(n)$. So the primary noise estimate $\hat{d}(n) \approx$ $d(n), \quad$ if $\hat{s}(z) \approx s(z)$ [18]. Where $W(z)$ is the control filter and $\hat{s}(z)$ is the secondary-path estimation of the secondary path $s(z)$. So the feedback active noise control system has two loops, adaptive 
algorithm loop, and controller loop. The interface among these two loops obscures the analysis of the system. So the feedback system can be modeled as feedforward system by assuming the perfect estimation of the secondary path. In $[18,27]$ an extensive frequency domain analysis was carried out and assumed that the secondary path estimate is perfect, therefore eliminating the reference signal synthesis error. Later, Bjarnason suggested the step size bound for broadband input and stochastic analysis of feedforward active noise control system [28].

The steady state weight update analysis of the adaptive filter for feedback active noise control system was presented by Sakai [29]. The convergence behavior of the feedback active noise control system with the secondary path estimation modeling error and imperfect secondary path estimation between the desired and synthesized reference signal are presented in [30].

\subsection{Hybrid ANC}

The hybrid system is the third type of ANC system, and it is a combination of both feedforward and feedback ANC systems. The feedforward part of a hybrid system is used to evaluate and cancel the noises, correlated with the reference signal. While the feedback part of a hybrid ANC system only deals with the uncorrelated noises [30-33]. The principle of hybrid ANC shown in Figure 4.

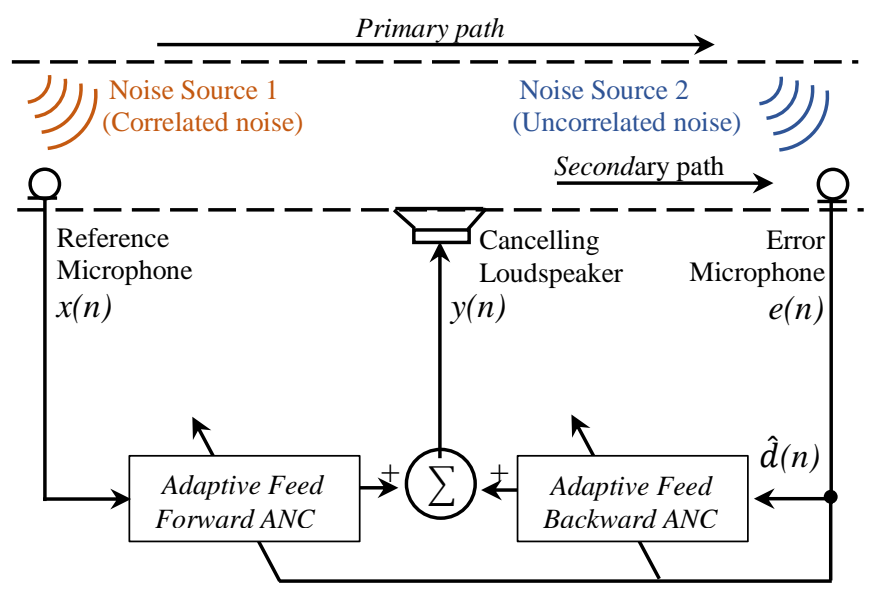

Figure 4. Principle of Hybrid Active Noise Control.

The secondary signal $y(n)$ is generated by using the output of both feedforward and feedback active noise control filter. As shown in Figure 4 it has two reference inputs: $x(n)$ from the reference sensor for feedforward controller and $\hat{d}(n)$, estimated reference signal for feedback controller. The hybrid system with lower order of FIR or IIR filters has low computational complexity and achieves the same performance as feedforward and feedback ANC system alone. Therefore, hybrid ANC is more suitable for a large number of broadband and narrowband noise canceling applications [34, 35]. An adaptation process of the hybrid system can further be increased by the modified hybrid model as in [36].

\section{POINT TO ZONE ANC}

In point to zone ANC, the noise source is localized, and the required quiet zone is spatial. The best approach to mitigate the noise is to embed the ANC system in the noise source application (i.e., engines, turbines, fan, and vacuum pump) or at least placed it very closely. Narrowband ANC and $1 \times 2 \times 2$ multi-channel ANC are the best suitable applications for this category.

\subsection{Narrowband ANC}

The system employed for canceling the periodic noises is called the narrowband active noise control system. In applied applications, this noise produced by rotating (or recurring) machinery for instance engines, is mostly periodic that comprises various narrowband components at a fundamental frequency and its harmonics. Single channel ANC system uses the non-acoustic sensors to cancel these narrowband components. These sensors like, accelerometer and tachometers synchronize internally generated sinusoidal reference signal to detect the fundamental frequency of the primary noise. The block diagram of narrowband active noise control using FXLMS algorithm is shown in Figure 5. Where $x(n)$, an internally synthesized reference signal is processed by an adaptive filter to cancel the primary noise. $\hat{s}(\mathrm{z})$ is the secondary path model of the secondary path $\mathrm{s}(\mathrm{z})$ and $e(n)$ is feedback to the adaptive filter for the adaptation process.

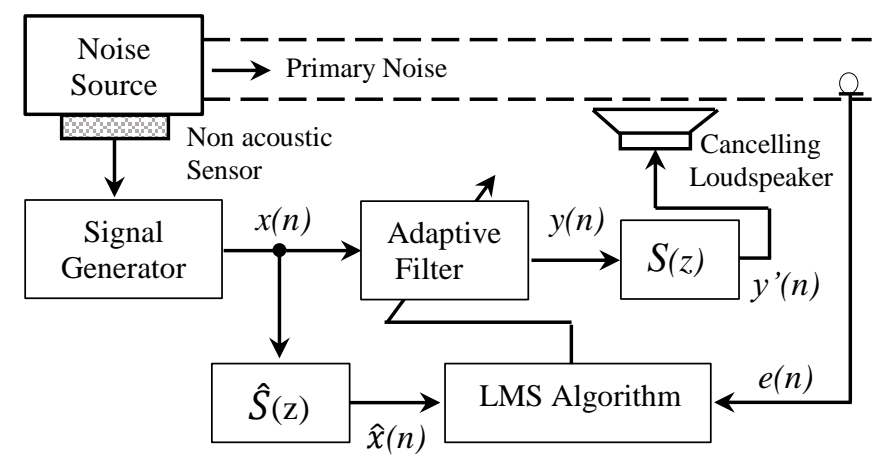

Figure 5. Narrowband Active Noise Control.

The frequency of internally generated primary noise is different from the actual primary noise due to the time varying characteristics of the noise sources. This frequency difference between primary noise and an internally generated reference signal is called frequency mismatch in narrowband active noise control system. The just $1 \%$ of frequency mismatch can severely degrade the performance of narrowband active noise control system [37]. The derivation of increase in the mean square error MSE caused by frequency mismatch is presented [38]. The controller's steady-state transfer function from the primary noise to the residual noise is used to derive the decrease in noise reduction caused by frequency mismatch [39]. The modified FxRLX was suggested to resolve this problem [40]. For narrowband ANC more convergence analysis of complex LMS and FxLMS has been presented in [41, 42]. The effect of frequency mismatch in narrowband active noise control has been investigated in [38, 43-45]. A complete theoretical analysis which shows that noise reduction and the convergence rate are the functions of frequency mismatch, phase error and step size in the secondary path model of the FxLMS algorithm is presented in [46].

The adaptive notch filter has the capability of tracking the timevarying frequency of the narrowband interferences by using an internally generated reference signal and it offers easy control of the bandwidth [47]. When sinusoid drifts slowly in frequency, an adaptive notch filter is beneficial [18]. The use of single frequency adaptive notch filters for active noise control application has been suggested in [48]. An idea of system connects multiple adaptive notch filters in parallel for attenuating periodic noise has been proposed by Ziegler in [49]. Analytically it is proved that the function of the single error signal to impart all adaptive filters 
with input signals initiates the extra tonal component in the residual noise.

The steady state performance is improved by using the bandpass filters to split the full-band error signal into multiple band limited sub-band error signals according to frequencies of reference signals [50]. The convergence rate of parallel narrowband active noise control is enhanced by using the delay less filter-bank to segregate the full-band excitation and error signals [51].

\subsection{Multichannel ANC}

Sparsely distributed noise source components in a large dimension duct, or in an enclosure of an industrial noise field have more complicated than the single channel point to point ANC, so it is necessary to use a multichannel active noise control system. Some of the best-known applications of a point to zone MCANC systems are impulsive and tonal noise in automobiles [52, 53], propeller-induced noise in helicopter/plane cabins [54, 55] and heavy moving machinery on a construction site [56]. More than one error sensor is needed to achieve a noise reduction in a big enclosure and automobile cabin. The simplest MCANC system has single reference sensor with $\mathrm{M}$ number of secondary sources and $\mathrm{N}$ number of error sensors as shown in Figure 6 [18].

$$
\begin{aligned}
& y_{1}(n)=\mathbf{w}_{1}^{T}(n) \mathbf{x}(n) \\
& y_{2}(n)=\mathbf{w}_{2}^{T}(n) \mathbf{x}(n)
\end{aligned}
$$

Where $y_{1}(n)$ and $y_{2}(n)$ are cancelling signals generated by adaptive filter $W_{l}(z)$ and $W_{2}(z)$ respectively. $P_{l}(z)$ and $P_{2}(z)$ are the primary paths and $S_{11}(n)$ and $S_{21}(n)$ are the secondary path from $y_{1}(n)$ to two error sensors, and $S_{12}(n)$ and $S_{22}(n)$ are the secondary path from $y_{2}(n)$ to two error sensors. Where $e_{1}(n)$ and $e_{2}(n)$ are the error signals from error sensors.

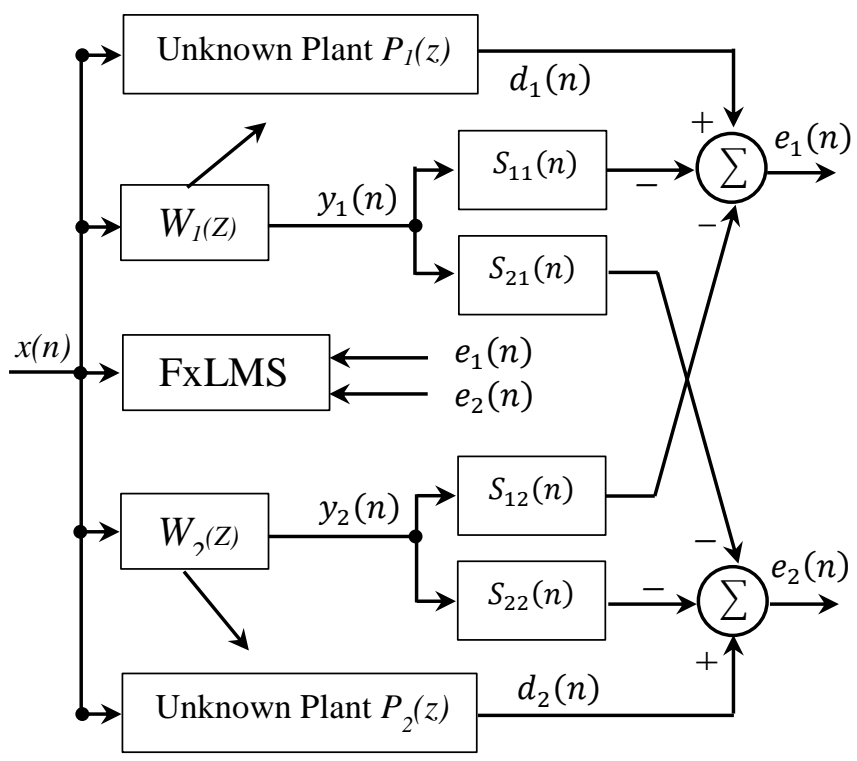

Figure 6. 1x2x2 Multichannel Feedforward ANC.

The computational complexity of MCANC system increases significantly, as the corresponding MC-FxLMS consist of $\mathrm{M}$ adaptive filters and $\mathrm{M} * \mathrm{~N}$ number of secondary path models.

In MCANC the location of each error sensor plays an important role for proper estimation of total acoustic energy [57]. A practical approach is, to use the secondary source close to the primary noise source [18]. In last one decade, many techniques and algorithms have suggested more efficient realization of MCANC system, of which Affine Projection Algorithm [58, 59], MC-RLS [60], wave field synthesis [61], and higher-order ambisonics [62] are famous for achieving spatial sound field control. The 2D higher-order ambisonics control, sparse FXLMS, and optimized time difference based reference microphone techniques are more advanced methods for the scattered sound field $[63,64]$.

\section{ZONE TO POINT ANC}

Zone to point active noise control is the case, when noise source is distributed everywhere (i.e., offices, bedrooms, airplanes, marketplaces) and the targeted quiet zone is local. Active headphones, ANC headrest, ANC pillow, ANC helmet, and ANC infant incubator are the classical examples of a zone to point active noise control.

\subsection{ANC Headphones}

Wearable listening devices, such as ANC headphones are popular and commercially most successful audio integrated ANC product [65]. This idea was originated in 1950, but notable research was done in mid of 1970's and 1980's when Kyle, William's, and Amar granted their patents in 1976, 77, and 84 respectively [6668]. These patents escalate the research in ANC headphones, and first commercial ANC headphone was introduced in late 1980's $[69,70]$. The design of ANC headphone is straightforward, but it has some practical constraint. The reference microphone to pick the reference signal is placed on the outside of the ear cup of ANC headphones while the secondary source is positioned inside to make a quiet zone around the eardrum, and error microphone is used to pick the residual error. Physical implementation and evaluation of ANC headphones presented in [71, 72] and the concept of virtual microphones used in [73]. Conventional methods have some drawbacks when the range of the controller coefficients is too large it is difficult to obtain an optimal solution. Secondly, it is also important to determine the controller structure and number of poles and zeros in advance that provides both robustness and performance. Recently more promising mixedsensitivity $\mathrm{H} \infty$ synthesis \& H2-norm optimization method is proposed, in which poles and zeros are used to model the controller transfer function instead of a rational transfer function $[74,75]$.

\subsection{ANC Headrest}

Active noise control headphones are used to avoid the exposure to unwanted noises but wearing this kind of devices for a long time is still uncomfortable. To surpass this drawback is the major motivation to active noise control (ANC) headrest. The idea of the active headrest is quite old, Olson and May carried their research on local ANC by developing a feedback model of an electronic sound absorber [76]. ANC headrest with feedforward approach was proposed in [77, 78] but there are some difficulties in implementation since the noise source is unknown and the location is an open environment. Rofaely \& Elliot suggested the single input single output multi-channel $\mathrm{H} 2 / \mathrm{H} \infty$ feedback controller, but it requires double computational resources [79]. In 2003 M. Pawelczyk proposed multi-channel feedback algorithm for active noise control headphones [80]. However, his first model requires four secondary loudspeakers with a large structure which is impractical for practical applications. Later, he suggested modified algorithm with two secondary sources [81] which are sufficient to create a local quiet zone [82]. Recently, auspicious single channel CICO (combined input combined output) headrest with an adaptive feedback algorithm was proposed and evaluated by experimental setup [82]. 


\subsection{ANC Infant Incubator}

According to WHO report, more than 15 million babies have a premature birth in a single year, and this number is increasing [83] These premature Infants or preemies are cared and monitored in an infant incubators in Neonatal Intensive Care Unit (NICU) for till they are considered fully developed and healthy. Infant incubator is an enclosure with a transparent section to holds the baby within. Vital statistics of baby and other parameters, such as humidity, temperature, and oxygen supply are monitored by sensors and devices inside the incubator. High level of noises from these devices and other equipment of NICU such as fans, IV pumps and warning sounds can result in adverse health effects for an infant. Passive noise control methods are ineffective to mitigate low-frequencies, and they also have some implementation constraints. This heightens the need for active noise control for infant incubators. Single and multiple channel ANC for infant incubator is widely investigated in last one decade and imperforate development model presented in [84-87]. In [88, 89] Kuo suggested wireless communication and cry classification integrated ANC to provide a better understanding of vital statics and bonding opportunity of a baby in an incubator. Recently, wireless communication integrated $\mathrm{ANC}$ is further improved by using hybrid ANC [90].

\section{ZONE TO ZONE ANC}

Zone to zone is the most challenging area of active noise control as the noise is spatially distributed over the region and goal to achieve satisfactory noise reduction in a large area. Environmental noise exposure such as industrial noises and transportation infrastructures (railway station, highways, and airport) noises have been linked to myriad health risks. Passive noise control is not always a viable and economical solution to mitigate these multidimensional noises. In such conditions, noise field is very complicated, and multichannel active noise control is expected to remove multi-dimensional noises. MCANC has been studied from mid of the 20th century, in 1956, Conover developed three channel active-system to mitigate the noise radiated by a large transformer [91, 92]. Conover model is based on the manual adjustment of amplitude and phase later, Onoda and Kido developed an automated system for reducing the noise in large transformer [93]. In recent years MCANC algorithms and techniques have been widely investigated and implemented to limit the noise pollution at our homes, offices, and working environments. Noise reduction performance and computational complexity of MCANC is always a major challenge for largescale practice. Active Soft Edge (ASE) and Active Acoustic Shielding (AAS) [94] are new promising techniques to mitigate the noises in open window and large ventilations.

\subsection{Active Acoustic Shielding}

Active acoustic shielding is based on the principle of ANC to attenuate the noises passing through an open window. The concept of AAS was proposed and implemented by Murao \& Nishimura [95]. The basic model of AAS is a set of ANC cells in an array, and each cell has approximately co-located secondary speaker and error microphone controlled by feedforward FxLMS algorithm. Initially, AAS was implemented on a small open window with few cells and the performance of AAS with moving sound sources, multiple sound sources, and reflected sounds are extensively studied [95-97]. In a second \& third phase of a study, multi-channel M(1-1)L FxLMS algorithm was suggested to enlarge the size of AAS window. In M(1-1)L FxLMS each cell of AAS array is controlled by its own reference and all neighboring error sensors [98]. To overcome the computational issues of
MCANC a decentralized control with multiple control algorithm M(1,1, L)FxLMS, M(1-1)MFxLMS, M(1-1-1t)FxLMS were suggested [99]. The physical layout of ASS-MCANC system is shown in Figure 7.

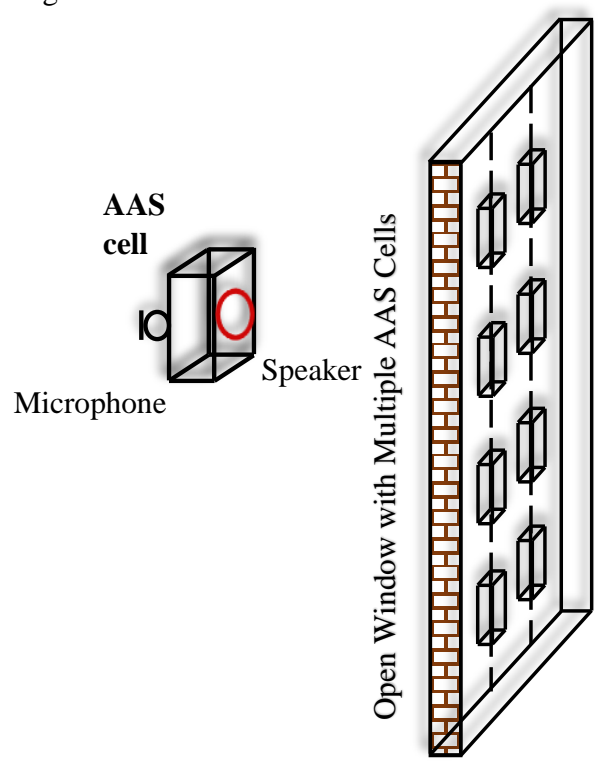

Figure 7. ASS-MCANC System for an Open Window.

The performance analysis of AAS windows for directional exterior noises was conducted in [100]. AAS for an open window is a new area of research, and physical limits of this technique are not clearly defined. Does AAS work for large zone/room? How many secondary noise sources are needed? Moreover, what is the ideal separation distance among these secondary noise sources? Lam B. answers all these questions by two-dimensional finite element modeling [101]. Recently, Fasciani conducted the comparative study of cone shape and flat shape speakers to minimize the obstruction caused by multi-channel ANC system in an open window [102]. However, in a practical implementation of AAS, the placement of error microphone is quite challenging and have some privacy concerns. These issues are surpassed by implementing the sound classification [103] and pre-adapted full rank fixed-filter model [104]. The use of error microphone is eliminated, and control filter is fixed for a various incidence of noises.

\section{CONCLUSION}

In this paper, we portray a physical classification of active noise control in four areas, on the basis of noise source and quiet zone characteristics. Those areas are point to point, zone to point, point to zone, and zone to zone. From this perspective, we comprehensively covered the progress within the last 80 years. However, we focused on introducing the recent research and development of ANC that have significant impacts on the performance of noise reduction in practical applications. In particular, we discussed some latest add-on features and presented a comprehensive study on multi-channel active acoustic shielding.

Despite the intensive research, we may conclude that all the four areas of ANC are open for further research. The latest concept of wave field synthesis, higher-order ambisonics, and parametric array loudspeakers for sparse field ANC still need further research to be practically adopted. 


\section{REFERENCES}

[1] (EEA), E.E.A., Noise in Europe 2014. 2014, (Publications Office of the European Union, 2014). (Publications Office of the European Union, 2014).

[2] Widrow, B. and S. Stearns, Adaptive signal processing. Englewood Cliffs, NJ, Prentice-Hall, Inc., 1985, 491 p., 1985.

[3] Haykin, S.S., Adaptive filter theory. 2002, Upper Saddle River, N.J.: Prentice Hall.

[4] Lee, K.-A., W.-S. Gan, and S.M. Kuo, Subband adaptive filtering: theory and implementation 2009: J. Wiley \& Sons.

[5] Elliott, S., Signal processing for active control. 2001: Academic press.

[6] Kuo, S.-M. and W.-S. Gan, Digital signal processors: architectures, implementations, and applications. 2005: Prentice Hall.

[7] Kuo, S.M., B.H. Lee, and W. Tian, Real-Time Digital Signal Processing: Implementations and Applications. 2nd ed. 2006: John Wiley \& Sons, Ltd.

[8] Gan, W.-S. and S.M. Kuo, Embedded signal processing with the micro signal architecture. 2007: J. Wiley \& Sons.

[9] Rayleigh, J.W.S.B., The theory of sound. Vol. 2. 1896: Macmillan.

[10] Guicking, D., Patents on Active Control of Sound and Vibration: An Overview. 2009: D. Guicking.

[11] Leventhall, H., A review on active attenuation and development of an active attenuator 'Open refuge'. 1988, W S Atkins Engineering Sciences: W S Atkins Engg. Sciences.

[12] Paul, L., Process of silencing sound oscillations. 1936, Google Patents.

[13] Kuo, S.M. and D.R. Morgan, Active noise control: a tutorial review. Proceedings of the IEEE, 1999. 87(6): p. 943-973.

[14] Burgess, J.C., Active adaptive sound control in a duct: A computer simulation. The Journal of the Acoustical Society of America, 1981. 70(3): p. 715-726.

[15] Kido, K. Reduction of noise by use of additional sound sources. in INTER-NOISE and NOISE-CON Congress and Conf. Proceedings. 1975. Institute of Noise Control Engg.

[16] Ross, C.F., A demonstration of active control of broadband sound. Journal of Sound Vibration, 1981. 74(3): p. 411-417.

[17] Nelson, P.A. and S.J. Elliott, Active control of sound. 1991: San Diego, CA: Academic.

[18] Kuo, S.M. and D. Morgan, Active noise control systems: algorithms and DSP implementations. 1995: John Wiley \& Sons, Inc.

[19] Hansen, C., et al., Active control of noise and vibration. 2012: CRC press.

[20] Roure, A., Self-adaptive broadband active sound control system. Journal of Sound and Vibration, 1985. 101(3): p. 429-441.

[21] Eriksson, L. and M. Allie, A practical system for active attenuation in ducts. Sound and Vibration, 1988. 22(2): p. 30-34.

[22] Morgan, D., An analysis of multiple correlation cancellation loops with a filter in the auxiliary path. IEEE Transactions on Acoustics, Speech, and Signal Processing, 1980. 28(4): p. 454-467.

[23] B. Widrow, D.S.a.S.S. On Adaptive Inverse Control. in Record of the Fifteenth Asilomar Conference on Circuits. 1981 November. Systems and Computers.

[24] Tabatabaei Ardekani, I. and W.H. Abdulla, Theoretical convergence analysis of FxLMS algorithm. Signal Processing, 2010. 90(12): p. 3046-3055.

[25] Ardekani, I.T. and W.H. Abdulla, On the stability of adaptation process in active noise control systems. The journal of the Acoustical Society of America, 2011. 129(1): p. 173-184.

[26] Ardekani, I.T. and W.H. Abdulla, Effects of Imperfect Secondary Path Modeling on Adaptive Active Noise Control Systems. IEEE Transactions on Control Systems Technology, 2012. 20(5): p. 1252-1262.

[27] Eriksson, L. Recursive algorithms for active noise control. in Proc. Int. Symp. Active Control of Sound Vibration 1991.

[28] Bjarnason, E., Analysis of the filtered-X LMS algorithm. IEEE Transactions on Speech and Audio Processing, 1995. 3(6): p. 504-514.

[29] Sakai, H. and S. Miyagi, Analysis of the adaptive filter algorithm for feedback-type active noise control. Signal processing, 2003. 83(6): p. 1291-1298.

[30] Wang, L.V., et al., Convergence Analysis of Narrowband Feedback Active Noise Control System With Imperfect Secondary Path Estimation. IEEE Transactions on Audio, Speech, and Language Process, 2013. 21(11): p. 2403-2411.

[31] Chen, S. and G.-P. Too, Simulations and experiments for hybrid noise control systems. Applied Acoustics, 2009. 70(2): p. 247-255.

[32] Xiao, Y. and J. Wang, A New Feedforward Hybrid Active Noise Control System. IEEE Signal Processing Letters, 2011. 18(10): p. 591-594.

[33] Akhtar, M.T. and W. Mitsuhashi, Improving performance of hybrid active noise control systems for uncorrelated narrowband disturbances. IEEE transactions on audio, speech, and language processing, 2011. 19(7): p. 2058-2066.

[34] George, N.V. and G. Panda, On the development of adaptive hybrid active noise control system for effective mitigation of nonlinear noise. Signal Processing, 2012. 92(2): p. 509-516.

[35] Kajikawa, Y., W.-S. Gan, and S.M. Kuo, Recent advances on active noise control: open issues and innovative applications. APSIPA Transactions on Signal and Information Processing, 2012. 1.

[36] Djigan, V.I., et al. Modified hybrid active noise control system. in 2015 IEEE East-West Design \& Test Symposium (EWDTS). 2015.

[37] Xiao, Y., L. May, and K. Khorasani. A robust narrowband active noise control system for accommodating frequency mismatch. in Signal Processing Conference, 2004 12th European. 2004. IEEE.

[38] Sakai, H. and Y. Hinamoto, An exact analysis of the LMS algorithm with tonal reference signals in the presence of frequency mismatch. Signal processing, 2005. 85(6): p. $1255-1262$. 
[39] Kuo, S.M. and S.P. Nallabolu. Analysis and Correction of Frequency Error in Electronic Mufflers using Narrowband Active Noise Control. in 2007 IEEE International Conference on Control Applications. 2007.

[40] Yegui, X., et al. A filtered-X RLS based narrowband active noise control system in the presence of frequency mismatch. in 2005 IEEE Int. Symp. on Circuits and Systems. 2005.

[41] Johansson, S., S. Nordebo, and I. Claesson, Convergence analysis of a twin-reference complex least-mean-squares algorithm. IEEE Transactions on Speech and Audio Processing, 2002. 10(4): p. 213-221.

[42] Chakraborty, M. and H. Sakai, Convergence analysis of a complex LMS algorithm with tonal reference signals. IEEE Transactions on Speech and Audio Processing, 2005. 13(2): p. 286-292.

[43] Hinamoto, Y. and H. Sakai, A Filtered-X LMS Algorithm for Sinusoidal Reference Signals\&mdash;Effects of Frequency Mismatch. IEEE Signal Processing Letters, 2007. 14(4): p. 259-262.

[44] Yegui, X., et al., Statistical properties of the LMS Fourier analyzer in the presence of frequency mismatch. IEEE Transactions on Circuits and Systems I: Regular Papers, 2004. 51(12): p. 2504-2515.

[45] Xiao, Y., et al., A New Robust Narrowband Active Noise Control System in the Presence of Frequency Mismatch. IEEE Transactions on Audio, Speech, and Language Processing, 2006. 14(6): p. 2189-2200.

[46] Jeon, H.J., T.G. Chang, and S.M. Kuo, Analysis of Frequency Mismatch in Narrowband Active Noise Control. IEEE Transactions on Audio, Speech, and Language Processing, 2010. 18(6): p. 1632-1642.

[47] Widrow, B., et al., Adaptive noise cancelling: Principles and applications. Proceedings of the IEEE, 1975. 63(12): p. 1692-1716.

[48] Elliott, S., I. Stothers, and P. Nelson, A multiple error LMS algorithm and its application to the active control of sound and vibration. IEEE Transactions on Acoustics, Speech, and Signal Processing, 1987. 35(10): p. 1423-1434.

[49] Ziegler Jr, E., Selective active cancellation system for repetitive phenomena. 1989, Google Patents.

[50] Yang, F., A. Gupta, and S.M. Kuo. Parallel multi-frequency narrowband active noise control systems. in 2009 IEEE Int. Conf. on Acoustics, Speech and Signal Processing. 2009.

[51] Chang, C.-Y., S.M. Kuo, and A. Siswanto. Enhanced offline secondary path modeling method for narrowband active noise control system. in Telecommunications and Signal Processing (TSP), 2016 39th Int. Conf. on. 2016. IEEE.

[52] Elliott, S., et al. The active control of engine noise inside cars. in INTER-NOISE Congress and Conference Proceedings. 1988. Institute of Noise Control Engineering.

[53] Sakamoto, K. and T. Inoue, Development of feedback-based active road noise control technology for noise in multiple narrow-frequency bands and integration with booming noise active noise control system. SAE Int. Journal of Passenger Cars-Mechanical Systems, 2015. 8(2015-01-0660): p. 1-7.

[54] Elliot, S., et al., In-flight experiments on the active control of propeller-induced cabin noise. Journal of Sound and Vibration, 1990. 140(2): p. 219-238.
[55] Kuo, S.M. and B.M. Finn, A general multi-channel filtered LMS algorithm for 3-D active noise control systems. Second Int. Con. on Recent Development in Air \& Structure Borne Sound and Vibration, Auburn AL, 1992: p. 345-352.

[56] Fahy, F. and J. Walker, Vehicle noise, in Adv. application in acoustics, noise and vibration. 2004, CRC Press.

[57] Kajikawa, Y., W.S. Gan, and S.M. Kuo. Recent applications and challenges on active noise control. in 2013 8th International Symposium on Image and Signal Processing and Analysis (ISPA). 2013.

[58] Bouchard, M. and S. Quednau, Multichannel recursiveleast-square algorithms and fast-transversal-filter algorithms for active noise control and sound reproduction systems. IEEE Transactions on Speech and Audio Processing, 2000. 8(5): p. 606-618.

[59] Bouchard, M., Multichannel affine and fast affine projection algorithms for active noise control and acoustic equalization systems. IEEE Transactions on Speech and Audio Processing, 2003. 11(1): p. 54-60.

[60] Hase, S., et al. Multi-channel ANC system using optimized reference microphones based on time difference of arrival. in 2015 23rd European Signal Processing Conference (EUSIPCO). 2015.

[61] Spors, S., R. Rabenstein, and J. Ahrens. The theory of wave field synthesis revisited. in 124th AES Convention. 2008.

[62] Ward, D.B. and T.D. Abhayapala, Reproduction of a planewave sound field using an array of loudspeakers. IEEE Trans. on Speech \& Audio Processing, 2001. 9(6): 697-707.

[63] Zhang, J., W. Zhang, and T.D. Abhayapala. Noise cancellation over spatial regions using adaptive wave domain processing. in Applications of Signal Processing to Audio and Acoustics, 2015 IEEE Workshop on. 2015.

[64] Zhangg, J., et al. Sparse complex FxLMS for active noise cancellation over spatial regions. in Acoustics, Speech and Signal Processing (ICASSP), 2016 IEEE International Conference on. 2016. IEEE.

[65] Chang, C.Y., et al., Listening in a Noisy Environment: Integration of active noise control in audio products. IEEE Consumer Electronics Magazine, 2016. 5(4): p. 34-43.

[66] Bose, A.G. and J. Carter, Headphoning. 1984, Google Patents.

[67] Kyle, G.L. and J.D. Hays Jr, Ear protection and hearing device. 1976, Google Patents.

[68] Williams, R.D., Hearing Protector, US Patent No. 4064362. 1977.

[69] Bartels, V. Headset with active noise reduction system for mobile applications. in Audio Engineering Society Convention 90. 1991. Audio Engineering Society.

[70] Sapiejewski, R. and J.J. Breen, High compliance headphone driving. 1993, Google Patents.

[71] Vu, H.S., K.H. Chen, and T.M. Fong. Active noise control for in-ear headphones: Implementation and evaluation. in 2015 IEEE International Conference on Consumer Electronics - Taiwan. 2015.

[72] Kuo, S.M., S. Mitra, and G. Woon-Seng, Active noise control system for headphone applications. IEEE 
Transactions on Control Systems Technology, 2006. 14(2): p. 331-335.

[73] Pawelczyk, M., Analog Active Control of Acoustic Noise at a Virtual Location. IEEE Transactions on Control Systems Technology, 2009. 17(2): p. 465-472.

[74] Liebich, S., et al. Active Noise Cancellation in headphones by digital robust feedback control. in European Signal Processing Conference. 2016.

[75] Liang, K.W. and J.S. Hu, An Open-Loop Pole - Zero Placement Method for Active Noise Control Headphones. IEEE Transactions on Control Systems Technology, 2017. 25(4): p. 1278-1283.

[76] Olson, H.F. and E.G. May, Electronic Sound Absorber. Journal of the Acoustical Society of America, 1953. 25(6): p. 1130-1136.

[77] Brothanek, M. and O. Jiricek. Formating of zones of quiet around a head simulator. in INTER-NOISE and NOISECON Congress and Conference Proceedings. 2002. Institute of Noise Control Engineering.

[78] Diego, M.d., A. Gonzalez, and C. Garcia. On the performance of a local active noise control system. in 1999 IEEE International Conference on Acoustics, Speech, and Signal Processing. Proceedings. ICASSP99. 1999.

[79] Rafaely, B. and S.J. Elliott, $\mathrm{H}_{2} / \mathrm{H}_{\text {infin }}$ active control of sound in a headrest: design and implementation. IEEE Transaction on Control Systems Technology, 1999. 7(1): p. 79-84.

[80] Pawelczyk, M., Multiple input-multiple output adaptive feedback control strategies for the active headrest system: Design and real-time implementation. Intl. Journal of Adaptive Control \& Signal Proc., 2003. 17(10): p. 785-800.

[81] Pawelczyk, M., Adaptive noise control algorithms for active headrest system. Control Engineering Practice, 2004. 12(9): p. 1101-1112.

[82] Siswanto, A., C.Y. Chang, and S.M. Kuo. Active noise control for headrests. in 2015 Asia-Pacific Signal and Information Processing Association Annual Summit and Conference, APSIPA ASC 2015. 2016.

[83] WHO | Preterm birth. WHO 2016 2016-11-18 15:20:52; Available from: http://www.who.int/mediacentre/factsheets/fs363/en/.

[84] Thanigai, P., S.M. Kuo, and R. Yenduri. Nonlinear Active Noise Control for Infant Incubators in Neo-Natal Intensive Care Units. in 2007 IEEE International Conf on Acoustics, Speech and Signal Processing - ICASSP '07. 2007.

[85] Liu, L., S.M. Kuo, and S. Gujjula. Development and applications of active noise control system for infant incubators. in 2009 IEEE International Conference on Systems, Man and Cybernetics. 2009.

[86] Liu, L., K. Beemanpally, and S.M. Kuo, Real-time experiments of ANC systems for infant incubators. Noise Control Engineering Journal, 2012. 60(1): p. 36-41.

[87] Kuo, S.M., L. Liu, and S. Gujjula, Development and application of audio-integrated ANC system for infant incubators. Noise Control Engineering Journal, 2010. 58(2): p. 163-175.

[88] Liu, L., K. Kuo, and S.M. Kuo. Infant cry classification integrated ANC system for infant incubators. in 2013 10th
IEEE International Conference on Networking, Sensing and Control (ICNSC). 2013.

[89] Liu, L. and S.M. Kuo. Wireless communication integrated active noise control system for infant incubators. in 2013 IEEE International Conference on Acoustics, Speech and Signal Processing. 2013.

[90] Liu, L., D. Lilin, and A. Kolla. Wireless communication integrated hybrid active noise control system for infant incubators. in 2016 IEEE Signal Processing in Medicine and Biology Symposium (SPMB). 2016.

[91] Eriksson, L.J., Waves of Silence. Digisonix, active noise control, and the digital revolution. 2015, Madison, WI 53718-8262 U.S.A.: Quarter Section Press. 13-20.

[92] Conover, W.B. and W.F. Gray, Noise reducing system for transformers. 1957, Google Patents.

[93] Onoda, S. and K. Kido. Automatic control of stationary noise by means of directivity synthesis. in Proc. 6th ICA Congress. 1968.

[94] Bhan, L. and G. Woon-Seng, Active Acoustic Windows: Towards a Quieter Home. IEEE Potentials, 2016. 35(1): p. 11-18.

[95] Murao, T. and M. Nishimura, Basic study on active acoustic shielding. Journal of Environment and Engineering, 2012. 7(1): p. 76-91.

[96] Huang, H., X. Qiu, and J. Kang, Active noise attenuation in ventilation windows. The Journal of the Acoustical Society of America, 2011. 130(1): p. 176-188.

[97] MURAO, T., et al., Basic study on active acoustic shielding (Improving noise-reducing performance in low-frequency range). Mechanical Engg. Journal, 2014. 1(6): p. EPS0065.

[98] MURAO, T., et al., Basic study on active acoustic shielding (Improving the method to enlarge the AAS window).

Mechanical Engineering Journal, 2016. 3(1): p. 15-0032215-00322.

[99] Murao, T., et al. Feasibility study on decentralized control system for active acoustic shielding. in INTER-NOISE and NOISE-CON Congress and Conference Proceedings. 2016. Institute of Noise Control Engineering.

[100] Kwon, B. and Y. Park, Interior noise control with an active window system. Applied Acoustic, 2013. 74(5): p. 647-652.

[101] Lam, B., et al., The physical limits of active noise control of open windows. 2015.

[102] Fasciania, S., et al. Comparative study of cone-shaped versus flat-panel speakers for active noise control of multitonal signals in open windows. in INTER-NOISE and NOISE-CON Congress and Conference Proceedings. 2015. Institute of Noise Control Engineering.

[103] Ranjan, R., et al. Selective Active Noise Control System for Open Windows using Sound Classification. in INTERNOISE and NOISE-CON Congress and Conference Proceedings. 2016. Institute of Noise Control Engineering.

[104] Bhan, L., et al. Feasibility of the full-rank fixed-filter approach in the active control of noise through open windows. in INTER-NOISE and NOISE-CON Congress \& Conference Proceedings. 2016. Institute of Noise Control Engg. 